\title{
Dinamizando el proceso de enseñanza-aprendizaje de la química. Las demostraciones químicas como una opción para cambiar la forma tradicional de enseñar química
}

\author{
Dynamizing the chemistry teaching-learning process. Chemical demonstrations as an \\ option to change the traditional form to teach chemistry
}

Manuel Fredy Molina Caballero, José Carriazo Baños y Diana Farías Camero

Universidad Nacional de Colombia, Profesores Departamento de Química Grupo de investigación en enseñanza de la química. mfmolinac@unal.edu.co, jcarriazog@unal.edu.co , dmfariasc@unal.edu.co

\section{Resumen}

Creemos que la "quimifobia" que existe a nivel social está generada en buena medida por la forma cómo se realiza la enseñanza de la química en las aulas. Para generar un cambio en las metodologías habituales proponemos, en este trabajo, el uso de las demostraciones químicas, mostrando un ejemplo a partir de la descomposición del peróxido de hidrógeno, buscando la forma de incorporar las demostraciones en el aula y considerando su carácter atractivo, la participación del estudiante, su vínculo conceptual y las cuestiones de seguridad a tener en cuenta. Dejamos a los profesores la propuesta, esperando que con su ayuda quizá podamos dar un toque especial a la enseñanza de la química.

\section{Palabras clave}

Demostraciones químicas, didáctica, enseñanza de la química, trabajo práctico.

\section{Abstract}

We consider that the "chemi-phobia" existing in the people is commonly generated by the manner like the teaching of chemistry in the dassroom is made. To produce a change on the routine methodologies we propose in this work the use of chemical demonstrations, showing an example from the hydrogen peroxide decomposition, looking for incorporation of dassroom demonstrations, and taking into account their attractive character, the student participation, the conceptual linkage, and the safety issues to take in mind. We deliver this proposal to the teachers, and we hope for your contribution to perhaps give a special "touch" to the teaching of chemistry.

\section{Keywords}

Chemical demonstrations, science education, chemisty teaching, practical work.

\section{Introducción}

La química es la ciencia de lo microscópico, que da explicaciones utilizando modelos atómicos que coinciden con lo que vemos en el mundo palpable, lo aul la convierte en una ciencia elegante y sorprendente. Aún así la química, según la concepción de la población que no ha tenido contacto con la química, es "riesgosa", y está ligada a la contaminación atmosférica, a los desechos químicos y a os contaminantes. En muchas ocasiones, es tan mal vista la química, que se asocia con algo nocivo cuando de alimentos se trata, pues muchos se venden con frases como "libre de químicos" o "sin tratamientos químicos". Sin embargo, quienes estudiamos la química sabemos que en todo lo que nos rodea hay productos químicos (naturales o artificiales) y eventualmente "fenómenos 
químicos", ...Desde que nos levantamos, y aún durmiendo, la química tiene su importancia de manera práctica: en la crema dental, en el jabón de manos y lavaplatos, en el pan, en el café, en la ropa, en los billetes, en la transformación de los alimentos en energía o en la de los combustibles en energía que mueve maquinas. . La química es la ciencia central.

La idea negativa que mucha gente ha creado sobre la química, quizá haya sido impulsada por la forma como ésta se enseña, sobre todo en los colegios donde la intención principal es cubrir contenidos y preparar a los estudiantes para el posible ingreso a la universidad, generando un aprendizaje descontextualizado y con poco impacto en la vida de las personas. Es sorprendente la gran semejanza en la presentación de los contenidos diseñados por muchas instituciones de educación secundaria en Colombia, con lo que se ve en un primer curso universitario de química. Muchas veces se concibe la idea de que aprender química es sólo aprender las partes formales de la química, como una estructura organizada de conceptos, esto es: estructura atómica, nomendatura, estequiometría, gases, disoluciones, equilibrio químico, etc., dejando de lado la relación de la química con cada asunto de nuestra vida, es decir la "química cotidiana": la química de los alimentos, de los medicamentos, del agua, de los combustibles, del aire, de los venenos, de los esteroides, etc.

La química debe ofrecer, para todos los estudiantes en general, una oportunidad maravillosa y fascinante de abordar la naturaleza y el mundo que los rodea, por ello las nuevas generaciones necesitan percibir la química de forma interesante, que despierte intriga y perplejidad, que se aprenda acercándose al hecho de hacer química mediante la generación intuitiva de interrogantes frente a hechos reales que motivan instantáneamente a la búsqueda de explicaciones, que de hecho fortalecen el desarrollo de competencias argumentativas y propositivas. De otro lado, dado que en las instituciones de secundaria no necesariamente se tienen laboratorios sofisticados para la química (como ocurre en universidades 0 centros tecnológicos dedicados a enseñar química) debemos recurrir a la creatividad y aun cambio de actitud en nuestra forma de enseñar química, y esto puede ocurrir con las demostraciones, una buena forma de enseñar química de forma dinámica, divertida, que permite romper con la rutina y generan inquietudes, que atraen e involucran a los alumnos en un aprendizaje activo y visual-real.

¿Qué son las demostraciones químicas?

Las demostraciones químicas constituyen una de las diversas actividades posibles en dase, donde se desarrolla un experimento dirigido totalmente por el profesor, son experiencias que generan impacto, incertidumbre y emoción en los estudiantes; además son actividades que sirven para aprender por asociación, el estudiante asocia la demostración con el concepto que el profesor trate de ilustrar. Son una forma de enseñar deleitando, para ilustrar leyes y principios (Roth et al., 1997). Evidentemente, las demostraciones químicas deben ser muy bien planeadas por el profesor, teniendo en cuenta todas las normas de seguridad necesarias y asociando el "experimento" a un conjunto de ideas previas normalmente generadas dentro de la temática en discusión, 0 aprendidas en cursos anteriores, para que pueda existir una "comunicación" entre la demostración y la estructura conceptual del individuo. Adicionalmente, posteriormente a la realización de la demostración en el aula, el profesor debe generar un espacio para el debate, en donde los estudiantes discuten los detalles del "experimento" observado, describen el fenómeno y proponen posibles explicaciones.

\section{Uso de las demostraciones químicas}

Las demostraciones en química tienen un valor didáctico enorme, porque motivan al estudiante a involucrarse en la clase y le proporcionan la oportunidad de tener un contacto real y cercano con la química, principalmente a aquellos estudiantes que quizás nunca la tendrán en su vida. Muchos reactivos y materiales usados en las demostraciones químicas son de origen casero o se obtienen fácilmente en las tiendas o lugares de ventas de productos químicos, son materiales y reactivos de baja calidad con lo cual disminuyen los costos. Igualmente, las demostraciones ayudan al docente a 
equiparse logística y conceptualmente para su dase, haciendo que él tenga su propio "kit" de demostraciones, que prepare técnicamente el manejo de reactivos y materiales de uso químico y que genere un marco teórico-práctico muy bien estructurado.

Las demostraciones químicas ayudan a mejorar la compresión de los textos, conduciendo a nuevas preguntas y despertando el interés por conceptos que, aún con la mejor ilustración en un libro, no cobran sentido para el estudiante. Las demostraciones generan vínculos entre los conceptos previos y los conceptos aprendidos en la dase, siendo esto una oportunidad para que el profesor pueda "graduar" el énfasis de la demostración con el objeto de maximizar las relaciones de interés (George, 1996).

Por otra parte, las demostraciones químicas ayudan a los profesores a darle mayor valor a la dase, despertando interés en los estudiantes por la actividad desarrollada; ayudan a potenciar las habilidades de observación, de pensamiento crítico y de análisis; conducen a indagar y generan impactantes oportunidades de aprendizaje. Esto es especialmente importante para aprender principios abstractos de la química, e implica relaciones entre el marcado simbolismo de la química, los fenómenos reales y las representaciones matemáticas. Las demostraciones dinamizan la dase, promueven el trabajo en el aula, generan emociones, despiertan la creatividad y generan un sentido de apropiación de conocimiento sobre el "misterio" de las cosas. Además, el estudiante siempre estará a la expectativa sobre las nuevas actividades sorprendentes: "choy qué traerá el profesor a la clase?". También debe resaltarse que, las demostraciones en el aula poseen la flexibilidad necesaria para acomodarse a los diferentes estilos de aprendizaje (Martin-Hansen, 2002).

\section{Desarrollo}

La planificación de las demostraciones químicas es importante en la medida en que confiere seguridad al trabajo y ayuda a lograr los objetivos propuestos. Algunas consideraciones importantes para tener en cuenta son (Collette, 1973):

Hacerlas en el momento apropiado. Buscar la relación entre el concepto a enseñar y la demostración utilizada. Deben ser ensayadas con anterioridad hasta que se logre una práctica segura y apropiada.

Deben ser visibles. Si una demostración no puede apreciarse generará frustración en los estudiantes, por ello es aconsejable hacerlas a gran escala.

Simples y sin complicaciones. Para ello debe ensayarse y buscar que lo necesario sea fácilmente obtenido.

Directa e impactante. No debe ser demasiado larga para no disminuir la atención del estudiante.

Se sugiere hacer una lista de demostraciones relacionadas con los tópicos a enseñar. En la web se pueden encontrar diversas páginas que incluyen demostraciones (ver: http://uww.chem.leeds.acuk/delights/texts), y el Joumal of Chemical Education publica continuamente experimentos sobre demostraciones (Tested demonstrations, In the dassroom), 0 en general si se coloca en un buscador la palabra "demostraciones químicas" surgen gran cantidad de sitios.

Participación del estudiante

En las demostraciones químicas, el estudiante no debe ser simple espectador, debemos dar al estudiante la oportunidad de describir y explicar con sus propias palabras las observaciones 
realizadas. Esto puede ser posible con la elaboración de un pequeño informe escrito sobre la demostración, en el aual debe:

Sustentar con fundamento teórico la demostración realizada. Esto es, explicar de manera coherente el evento observado, empleando los conceptos y principios involucrados en éste.

Realizar un dibujo del montaje experimental, induyendo la utilidad de cada parte 0 reactivo.

Incluir los datos tomados o las variables sobre las cuales se dio importancia.

Elaborar unas condusiones sobre el efecto de la demostración en relación al concepto aprendido, así como induir unos comentarios que busquen mejorar la presentación y ponga en evidencia las ideas que se formaron con la demostración. Es posible que algunos estudiantes generen ideas erróneas ante las cuales el profesor debe estar atento para corregirlas.

Un ejemplo de una demostración química

Las demostraciones químicas en los colegios deben crear "misterio" o expectativa al comienzo, por eso la demostración presentada aquí puede iniciarse con la siguiente historia: "La química es tan importante y difundida que le sirve hasta a los elefantes dependen de ella y la utilizan. Ustedes saben que el elefante es el animal que posee el cerebro más grande y por ello algunos creen que posee cierta inteligencia.. miren que un cerebro grande no es sinónimo de inteligencia. El elefante en sus baños corporales, como es grande, utiliza cosas de tamaño gigante; entonces no utiliza un champú corriente, utiliza un champú especial. Un amigo mío que viajó a África, trajo un elefante y semanalmente lo baña en su casa en una tina gigante; él me solicitó que le fabricara un champú especial y eso es lo que deseo mostrarles...mi producto lo llamé "champú elefantero"... Bueno ese champú se prepara así..."

Lo que el profesor va a mostrar es la descomposición del peróxido de hidrógeno representada en la reacción

$$
\mathrm{H}_{2} \mathrm{O}_{2} \rightarrow \mathrm{H}_{2} \mathrm{O}+1 / 2 \mathrm{O}_{2}
$$

Esta reacción es lenta a condiciones normales, sin embargo auando se utiliza el ion yoduro el proceso es rápido. El efecto del catalizador puede comprenderse con el siguiente esquema de reacciones:

$$
\begin{aligned}
& \mathrm{H}_{2} \mathrm{O}_{2(\mathrm{ac})}+\mathrm{I}_{(\mathrm{ac})}^{-} \rightarrow \mathrm{H}_{2} \mathrm{O}_{(\mathrm{l})}+\mathrm{IO}_{(\mathrm{ac})}^{-} \\
& \mathrm{H}_{2} \mathrm{O}_{2(\mathrm{ac})}+\mathrm{IO}_{(\mathrm{ac})}^{-} \rightarrow \mathrm{H}_{2} \mathrm{O}_{(\mathrm{l})}+\mathrm{I}_{(\mathrm{ac})}^{-}+\mathrm{O}_{2(\mathrm{~g})}
\end{aligned}
$$

$$
2 \mathrm{H}_{2} \mathrm{O}_{2(\mathrm{ac})} \rightarrow 2 \mathrm{H}_{2} \mathrm{O}_{(\mathrm{l})}+\mathrm{O}_{2(\mathrm{~g})}
$$

El ion yoduro actúa como catalizador porque inicialmente se consume y luego se genera nuevamente en el mismo proceso, y como consecuencia modifica la velocidad de la reacción, haciéndola más rápida.

Materiales y reactivos

NaI o KI, disolución saturada.

Peróxido de hidrógeno entre el 30-50\% de concentración.

Champú para alfombras.

Probeta gigante (o un florero).

Un erlenmeyer de $250 \mathrm{~mL}$

Bombas (globos).

Procedimiento 
Los reactivos se preparan y se pueden colocar en recipientes plásticos. El yoduro y el peróxido de hidrógeno se consiguen en una tienda de productos químicos, hay muchas en el directorio telefónico. El peróxido de hidrógeno comercial lo venden por galones a precios económicos, con lo aual aseguramos el reactivo para realizar la demostración muchas veces.

A unos $100 \mathrm{~g}$ de $\mathrm{NaI}$ se pueden adicionar $100 \mathrm{~mL}$ de agua de la llave. El peróxido de hidrógeno debe manipularse con guantes y gafas, ya que a esa concentración puede provocar quemaduras. Se debe evitar cualquier contacto con la piel, y en caso de presentarse debe lavarse el área afectada con abundante agua. Para mayor información debe consultarse su ficha de seguridad, mediante un buscador de Intemet (colocar las palabras ficha de seguridad y el nombre de la sustancia, peróxido de hidrógeno) o en un manual de fichas técnicas (Toxic and Hazardous, Industrial Chemical Safety Manual).

En la siguiente seauencia de fotografías se ilustran los pasos a seguir.

Los reactivos, se pueden llevar en una especie de "kit" y se deben cargar en frascos plásticos debidamente marcados.

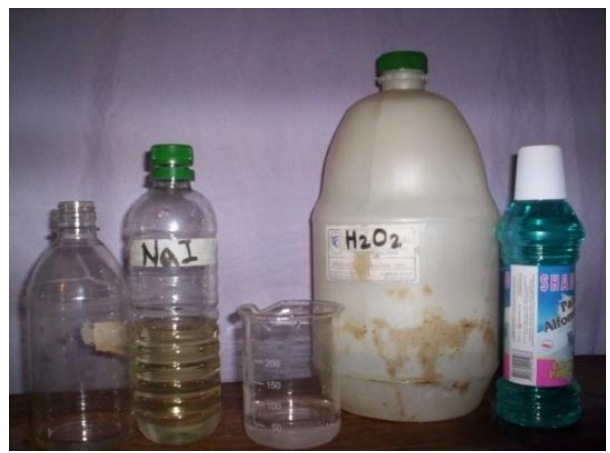

Figura 1. Reactivos empleados en la demostración.

Para demostrar que la reacción de descomposición del peróxido de hidrógeno produce oxígeno podemos hacer una pequeña prueba en un frasco plástico. Colocamos unos $20 \mathrm{~mL}$ de disolución de $\mathrm{NaI}$, adicionamos otros $20 \mathrm{~mL}$ de peróxido de hidrógeno (aquí al 50\%) y colocamos rápidamente una bomba en la boca del frasco. Como vemos en la siguiente imagen (figura 2) se produce un burbujeo rápido que llena la bomba de oxígeno. Luego, con cuidado soltamos la bomba y permitimos la salida lenta del oxígeno sobre la llama de una vela encendida, con lo cual la llama se aviva notoriamente. Aquí se puede ampliar la disausión hablando del papel del oxígeno en las combustiones y sobre su porcentaje en la atmósfera. También podemos hacer que algunos estudiantes toquen el frasco, que se calienta apreciablemente, y discutir sobre las reacciones exotérmicas, en general sobre los cambios energéticos en una reacción química.

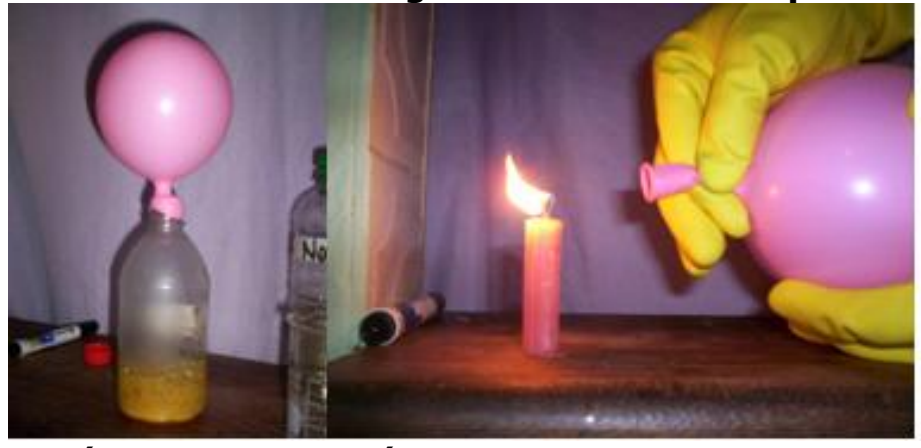

Figura 2. Reacción de descomposición del $\mathrm{H}_{2} \mathrm{O}_{2}$ y la evidencia de la presencia de $\mathrm{O}_{2}$

Luego, realizamos la reacción en la probeta gigante (o en un florero que se puede comprar en 
un almacén de productos químicos). En la figura 3 vemos el orden de adición de los reactivos: 50 $\mathrm{mL}$ de $\mathrm{NaI}, 30 \mathrm{~mL}$ de champú. Agitamos y luego adicionamos $50 \mathrm{~mL}$ de peróxido. La reacción se produce inmediatamente. Aquí, a diferencia del trabajo con el globo presentado anteriormente, se produce espuma, por la presencia del champú, que atrapa las burbujas de oxígeno generando "pompas de jabón".

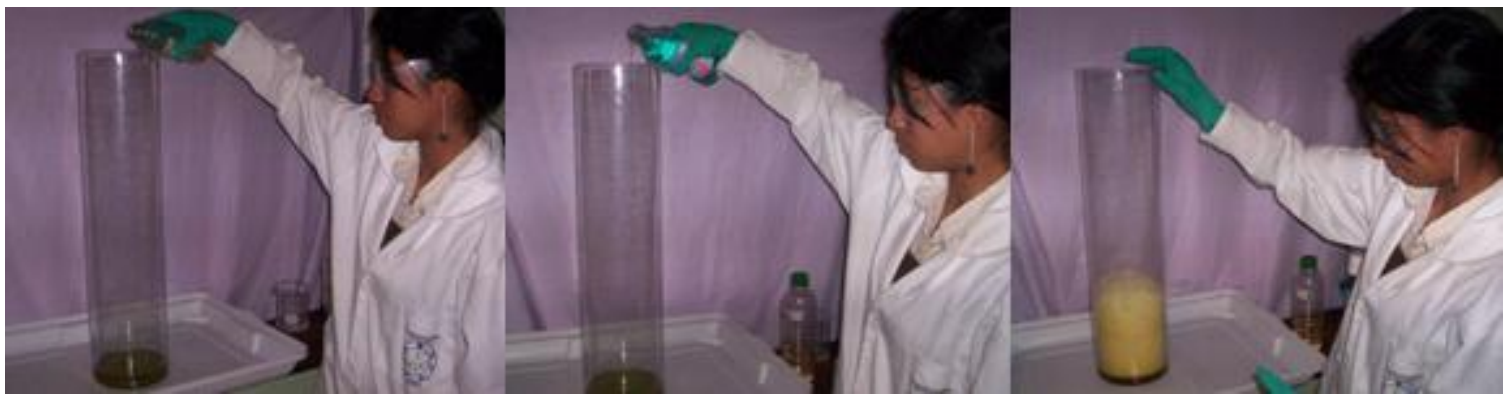

Figuras 3. Adición de reactivos y reacción.

La espuma se produce rápidamente y sube por la probeta, eso crea un espectáculo para los estudiantes. A medida que se adquiere práctica, se aprende a dosificar la cantidad de cada reactivo para lograr que la espuma se produzca más o menos rápido. Es bueno contar con un recipiente (aubeta) debajo de la probeta, para retirar con mayor facilidad los desechos. La espuma está caliente, si algún alumno desea tocarla debe utilizar guantes. Aquí podemos completar la historia del champú para elefante, pues definitivamente sí produce bastante espuma.

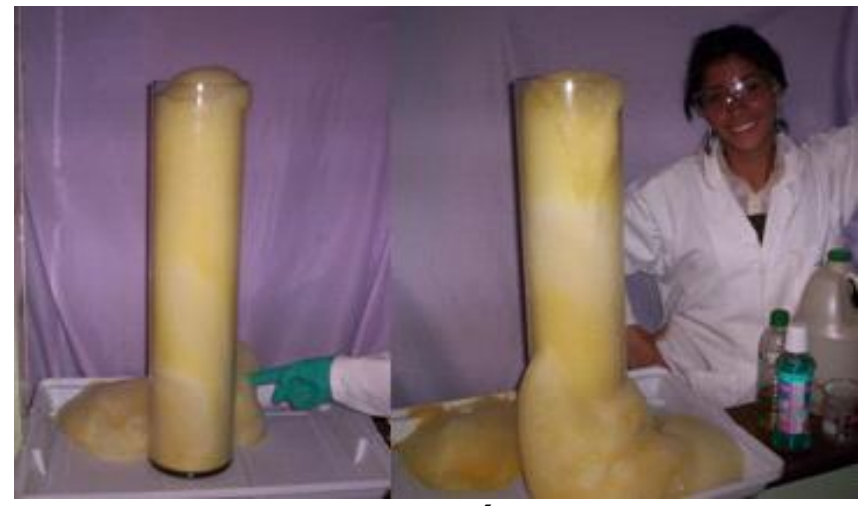

Figura 4. El champú del elefante.

Esta actividad, una vez se conoce y maneja, es tan fácil que hasta un niño la puede realizar (sin embargo, aconsejamos que ningún niño maneje el peróxido de hidrógeno sin la supervisión de un profesor de química- profesional en el área). Confiamos que el docente se anime y reflexione sobre otras implicaciones de la demostración en su aula de dase.

Los residuos generados se pueden disponer por el desagüe con abundante agua, pues los productos generados no son nocivos (agua, oxígeno, iones sodio y yoduro).

\section{La demostración y su vínculo conceptual}

Los temas que se pueden ilustrar con esta demostración pueden ser: estequiometría, el papel de los catalizadores con su concepto más simple sobre la no desaparición en una reacción, o con su concepto más elaborado sobre la modificación de la secuencia de la reacción química, aumentando su velocidad. Puede repetirse la demostración por adición de otra cantidad de peróxido, con lo cual la reacción se repite, sin la adición de más yoduro, pues éste no se ha gastado en el proceso. Desde aquí, se puede estudiar la representación de las reacciones sobre la coordenada de reacción, mostrando los efectos del catalizador sobre la energía de activación; también se puede asociar con 
los catalizadores biológicos, las enzimas, y con la importancia de la catálisis en procesos como la obtención de amoniaco para elaborar fertilizantes o en la refinación del petróleo para obtener gasolina y sus derivados.

Obviamente, se puede discutir sobre las reacciones químicas, su representación simbólica y su hecho real; o sobre la producción de oxígeno y la producción de pompas de jabón. En fin, el docente puede conduair la demostración como mejor lo considere respecto al desarrollo de su aurníaulo. Adicionalmente, mediante la reacción de descomposición del peróxido de hidrógeno, puede abordarse la discusión sobre su potencial oxidante y por tanto su empleo en procesos de impacto ambiental: en la descontaminación de aguas residuales que contienen compuestos orgánicos tóxicos, sobre lo cual existe mucha literatura científica para consultar (umw.sciencedirectcom).

Algunas consideraciones de seguridad

Es importante conocer exactamente qué se va a hacer; muchos accidentes ocurren porque el profesor o los estudiantes ignoran qué peligros puede tener el uso de determinados reactivos. Según Summerlin et al. (1987), se requiere:

1. Conocer las propiedades de los productos químicos (fichas técnicas) y las reacciones involucradas en todas las demostraciones.

2. Llevar protección apropiada, gafas, guantes y bata.

3. Comunicar a la audiencia sobre los peligros que se pueden presentar.

4. Evitar la generación de gases tóxicos, como $\mathrm{NO}_{2}, \mathrm{SO}_{2}$ y $\mathrm{H}_{2} \mathrm{~S}$.

5. Poseer un espacio seguro para colocar y llevar los reactivos.

6. No permitir que los espectadores toquen, manipulen o prueben las sustancias.

7. Poseer un procedimiento escrito (ya probado), información de peligrosidad y disposición de residuos.

\section{Consideraciones finales}

Los profesores necesitamos darle un "toque" especial a las dases de química, debemos salir de la rutina del tablero y del libro de texto (sin pretender abandonar estos elementos escolares), necesitamos dinamizar nuestras dases, y las demostraciones químicas confieren ese "toque" especial que le falta a la dase de química, ese "ingrediente" que permite enlazar algunas piezas como: conceptos-símbolos-ecuaciones-realidad, que son enormemente fundamentales en el "juego" del aprendizaje de la química. Además, somos responsables de llevar una imagen más real de la química y una imagen que perdure en la mente de nuestros estudiantes, somos agentes de cambio, en este caso de cambios de creatividad e innovaciones didácticas.

\section{Bibliografía}

Collette, A. T. (1973). Science Teaching in the Secondary Schoof; Allyn and Bacon: Boston, MA. 1-33.

George, B. K. (1996). Lecture Demonstrations, Past and Present. The Chemical Educator: 1 (5),

Martin-Hansen, L. (2002). Defining inquiry. The Saience Teacher: 69,34-37.

Roth, W.M.; McRobbie, C.J.; Lucas, K.B.; Boutonne, S. (1997). Why may students fail to leam from demonstrations?. A social practice perspective on learning in Physics. Joumal of Research in Science Teaching. 34, 509-533.

Summerlin, L R.; Borgford, C. L; Ealy, J. B. (1987). Chemical Demonstrations: A Sourcebook for Teachers, Vol. 2; American Chemical Society: Washington, DC. 